\title{
Absolute angle-differential elastic and vibrational excitation cross sections for electron collisions with tetrahydrofuran
}

\author{
M Allan \\ Department of Chemistry, University of Fribourg, Fribourg, Switzerland
}

\begin{abstract}
Absolute angle-differential elastic and vibrational excitation cross sections for electron collisions with tetrahydrofuran were measured in the energy range 0.1-20 eV, extending existing measurements to lower energies. The elastic cross sections were measured as a function of scattering angle from $10^{\circ}$ to $180^{\circ}$ at energies of $2 \mathrm{eV}, 6 \mathrm{eV}, 10 \mathrm{eV}$ and $20 \mathrm{eV}$, and as a function of electron energy at $45^{\circ}, 90^{\circ}, 135^{\circ}$ and $180^{\circ}$. The agreement with previous measurements and with the published theoretical work was generally satisfactory. The RamsauerTownsend minimum was observed at low energies, down to $0.24 \mathrm{eV}$ at $180^{\circ}$. Three additional minima were observed at $1.13,4.74$ and $15.3 \mathrm{eV}$ in the $180^{\circ}$ elastic cross section. Vibrational excitation cross sections are reported as a function of electron energy from the threshold to $16 \mathrm{eV}$. They reveal threshold peaks and broad bands at 6.2 and $10.8 \mathrm{eV}$, attributed to shape resonances, in agreement with theoretical predictions, although the calculated energies are generally somewhat higher. A broad enhancement of the $\mathrm{CH}_{2}$ scissoring vibration is observed around $2.6 \mathrm{eV}$, implying a low-lying (shape) resonance similar to that observed earlier in cyclopropane.
\end{abstract}

\section{Introduction}

The interest in electron-induced chemical processes at low energies has recently been renewed by the discovery that electrons at subionization [1] and even subexcitation energies [2] damage DNA. Electron interactions with tetrahydrofuran (THF) have been studied because it serves as a convenient model for the sugar ring in the DNA backbone [3].

Of particular importance in this respect are studies in the condensed phase. Lepage et al [4] studied resonances in THF by means of resonant vibrational excitation (VE) in the gas phase but primarily in thin-film THF and detected at least three resonances (i.e., temporary anion states), located near 4, 7.5 and $10 \mathrm{eV}$ in the frozen film. They observed a similarity with spectra of cyclopentane and concluded that the resonances in THF, revealed by VE, need not be attributed to the presence of the oxygen non-bonding electrons. Antic et al [3] studied 
the electron-induced yield of negative ions from frozen THF and observed the formation of $\mathrm{H}^{-}$at $10 \mathrm{eV}$ attributed to a core-excited resonance. Electron energy loss spectra (EELS), in particular the observation of a $\left(n, \pi^{*}\right)$ transition, revealed neutral degradation products containing carbonyl groups. Antic et al [5] reported a resonance at $23 \mathrm{eV}$ which decays into a highly excited state undergoing a further dipolar dissociation. The formation of aldehydes from THF frozen on a $\mathrm{Kr}$ substrate was studied in detail by Breton et al [6] and Jäggle et al [7] by means of vibrational and electronic EELS of the products. Strong rise of aldehyde production was observed from about $6 \mathrm{eV}$ and correlated to $\left(n, \sigma_{\mathrm{CO}}^{*}\right)$ electronic excitation threshold of THF, together with core-excited resonances around 9 and $10 \mathrm{eV}$. The absolute cross section reached a value of $\sim 0.6 \AA^{2}$ above $11 \mathrm{eV}$. A small feature found around $3 \mathrm{eV}$ was proposed to result from a $\sigma^{*}$ shape resonance. The formation of olefins and $\mathrm{CO}$ were identified in the later study. Electron trapping processes in condensed THF were studied quantitatively by Park et al [8]. The charge trapping cross section for $6-9 \mathrm{eV}$ electrons was found to have an upper limit of $0.004 \AA^{2}$, much smaller than the aldehyde production cross section mentioned above, leading to the conclusion that the major part of THF degradation has electronic excitation and not dissociative electron attachment (DEA) as the primary step. A much larger trapping cross section $\left(\sim 0.4 \AA^{2}\right.$ for two monolayers $)$, strongly dependent on the quantity of deposited THF, and proceeding via intermolecular stabilization, was found in the $0-0.1$ eV range.

The condensed phase work was complemented by gas-phase-DEA fragmentation studies performed by Sulzer et al [9] and in this laboratory [10]. Of particular importance is the quantitative study of Aflatooni et al [11] who found two DEA bands, at 6.2 and $8 \mathrm{eV}$, with a surprisingly small cross section, $1.5 \times 10^{-4} \AA^{2}$, about a factor 30 less than that in 3-hydroxy substituted THF.

Indispensable for the global understanding of the electron-induced processes are also gas-phase studies not directly involving chemical change. Zecca et al [12] and Możejko et al [13] measured the absolute total cross section for electron scattering by gas-phase THF (Zecca et al reported also the positron cross section). The elastic differential cross section (DCS) at $20 \mathrm{eV}$ and above was measured by Milosavljević et al [14]. Absolute elastic cross-sections in the energy range of $6.5-50 \mathrm{eV}$ and angular range between $10^{\circ}$ and $130^{\circ}$ were measured by Colyer et al [15], in the ranges $6-20 \mathrm{eV}$ and $20^{\circ}-180^{\circ}$ by Dampc et al [16]. Vibrational excitation cross sections have been measured very recently by Dampc et al [17].

Several high-level scattering calculations on THF have been reported. Trevisan et al [18] reported an ab initio calculation of the elastic differential and momentum-transfer cross sections using the complex Kohn variational method. Winstead and McKoy [19] calculated the elastic differential and momentum-transfer cross sections using the Schwinger multichannel method. Bouchiha et al [20] used the $R$-matrix method with Born correction to calculate the angle-integrated elastic cross section, the inelastic (for electronic excitation) cross section and the energies of a number of core-excited (Feshbach) resonances. Tonzani and Greene [21] calculated the integral elastic cross section and gained further insight into the resonant structure by considering time delay.

The present work reports measurements of elastic and VE cross sections over a wide angular range up to $180^{\circ}$ and at energies from about $0.1 \mathrm{eV}$ up to $20 \mathrm{eV}$. It extends the existing experimental work to lower energies.

\section{Experiment}

The measurements were performed using a spectrometer with hemispherical analysers $[22,23]$. The energy resolution was about $20 \mathrm{meV}$ in the energy-loss mode, corresponding 
to about $15 \mathrm{meV}$ in the incident electron beam, at a beam current of around $400 \mathrm{pA}$. The energy of the incident beam was calibrated on the $19.365 \mathrm{eV} \mathrm{[24]}{ }^{2} \mathrm{~S}$ resonance in helium and is accurate to within $\pm 10 \mathrm{meV}$. The instrumental response function was determined on elastic scattering in helium and all spectra were corrected as described earlier [22].

Absolute values of the cross sections were determined by the relative flow technique as described by Nickel et al [25] using the theoretical helium elastic cross sections of Nesbet [26] as a reference. The measurement of the relatively large and polar molecule poses additional challenges and the procedure will therefore be described here in more detail.

The sample and He flows were determined from the rate of pressure rise in a fixed closed manifold comprised of a stainless-steel test tube and the combined volumes of the Baratron gauge and the interconnecting tubes. Its volume is calculated from the volume of the test tube, the diameters and lengths of the interconnecting tubes and the Baratron gauge to be $66 \mathrm{~cm}^{3}$.

The determination of the sample flow from the pressure rise could, in principle, fail for a polar 'sticky' molecule such as THF, if a certain (pressure-dependent) fraction of the sample would be adsorbed on the inner surface of the inlet manifold as the pressure rises - this fraction of the flow would then not contribute to the pressure rise. An indication that the scheme of the flow measurement is valid stems from the fact that the pressure rise during the flow measurement is nearly exactly linear, so that if a significant number of the THF molecules were adsorbed, then the fraction of the adsorbed molecules would have to be exactly proportional to pressure. To exclude even this possibility, the flow was once determined in an alternate way, from the weight of the sample consumed over a given period of time $(80.8 \mathrm{mg}$ in $23 \mathrm{~h}$ and $35 \mathrm{~min})$. The result $\left(0.375 \mathrm{mbar} \mathrm{cm}^{3} \mathrm{~s}^{-1}\right)$ was in a good agreement with the value derived from the pressure rise $\left(0.362 \mathrm{mbar} \mathrm{cm}^{3} \mathrm{~s}^{-1}\right)$. The test was performed with a THF pressure behind the nozzle of 0.212 mbars. This method of flow determination is, however, impractical at lower pressures and it cannot be excluded that the sample adsorption becomes significant at substantially lower pressures.

The second potential problem could stem from the fact that the gas beam profile depends on the sample pressure. This effect was investigated in detail experimentally by Buckman et al [27]. As a consequence, the value of the cross section determined by the relative flow method depends on the pressure. To ensure equal gas beam profiles, Trajmar and co-workers [25] recommended to adjust the driving pressures behind the nozzle such that the mean free paths of the THF sample and the He reference are equal and are longer than the nozzle diameter. This condition is commonly applied by all research groups employing the relative flow method. Zubek and co-workers [16] discussed the gas kinetic molecular diameter of THF and derived the recommended value of the ratio of the driving pressures to be $P_{\mathrm{THF}}: P_{\mathrm{He}}=1: 4.6=0.217$.

The instrument used in the present study does not use a collimating capillary, but a nozzle shown on the right of figure 1. (The same nozzle was used also in all past absolute measurements using this spectrometer.) The gas is transported by a $4.3 \mathrm{~mm}$ inner diameter tube, the diameter is then reduced first to $1.2 \mathrm{~mm}$ and then to $0.6 \mathrm{~mm}$, for the last $2 \mathrm{~mm}$ of length, and finally expands to vacuum through a $d=0.25 \mathrm{~mm}$ diameter aperture in a material about $0.2 \mathrm{~mm}$ thick. The collimation effect of the capillary is largely absent in this scheme, but the profile of the gas beam is presumably closer to the cosine distribution, and less dependent on the pressure and the type of the gas. (The idea of using an aperture instead of a capillary (array) for the gas inlet to avoid the gas beam profile problem has recently been employed consequently by Khakoo et al [28].) Despite this aperture-like geometry of the nozzle, the value obtained for the cross section does depend on the sample pressure used as illustrated in the left part of figure 1 . The cross section values given in this paper refer to the pressure ratio recommended by Dampc et al [16], $P_{\mathrm{THF}}: P_{\mathrm{He}}=1: 4.6=0.217$, indicated by the vertical dashed line in figure 1. (In reality, it is difficult to adjust a given pressure exactly using a 


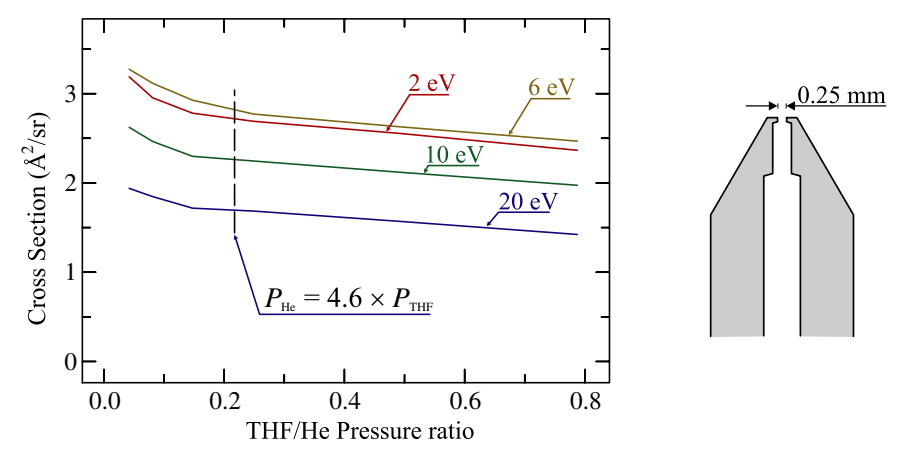

Figure 1. The dependence of the values obtained for the elastic cross section at $\theta=45^{\circ}$, at the four energies indicated, on the ratio of the sample and He pressures, obtained by comparing signals from helium at a pressure of 0.233 mbars with THF signals recorded with pressures ranging from 0.010 to 0.174 mbars. The cross sectional view of the nozzle is shown on the right.

regulating needle valve and the actual pressures deviated slightly from the ideal ratio, but the cross sections were corrected for the pressure dependence using a slope derived from the data in figure 1.) The sensitivity of the measured value on the sample pressure, derived from the curves in figure 1, is such that increasing (decreasing) the sample pressure by a factor of 2 changes the measured value by $-6 \%(+2.7 \%)$. This means that even deviations of a factor of 2 from the optimal pressure ratio are absorbed in the error limits of the present experiment, which are given as $\pm 15 \%$.

The mean free path of the THF molecules at 0.05 mbars, the condition indicated by the vertical dashed line in figure 1 , is about $\lambda=0.9 \mathrm{~mm}$, fulfilling (but not by far) the condition $\lambda \geqslant d$ recommended by Nickel et al [25].

Recording the helium and sample signals (at four energies) takes about $1-2 \mathrm{~h}$ each. The gas pressure is generally stable within $\pm 2 \%$, the electron beam current within $\pm 5 \%$ over this time period. The residual variations are corrected for by taking regular readings automatically and taking them into account in the summing procedure. The background was determined by recording the signal with either gas turned off, or gas introduced into the main chamber through a by-pass valve. It was subtracted when significant (mainly in the forward direction). The background pressure in the main chamber (uncorrected gauge reading) was nearly unmeasurable with a Penning gauge $\left(\leqslant 2 \times 10^{-7}\right.$ mbars $)$ during the absolute measurements, and about $2 \times 10^{-6}$ mbars during measurements of excitation functions and angular distributions. (The main chamber is pumped by a $400 \mathrm{~mm}$ and a $150 \mathrm{~mm}$ diameter diffusion pump in parallel, the compartment containing the electron energy analysers by another $150 \mathrm{~mm}$ diameter diffusion pump.)

The inelastic cross sections for the individual vibrations are much less precise because the vibrational frequencies are densely spaced and could not be fully resolved. The absolute values for the inelastic cross sections are therefore only indicative; they describe the intensity of the inelastic signal at a given energy loss, without attempts to deconvolute individual vibrational peaks. The total vibrationally inelastic cross section was obtained by integrating under the (response function corrected) energy-loss spectrum and comparing to the elastic signal. This total cross section is not affected by the peak overlap and is thus more precise, within about $\pm 25 \%$.

The angular distributions were measured using the combined mechanical setting of the analyser and magnetic deflection using the magnetic angle changer (MAC) [29, 30], correcting the curves for the instrumental response function, and fitting them to the discrete absolute 

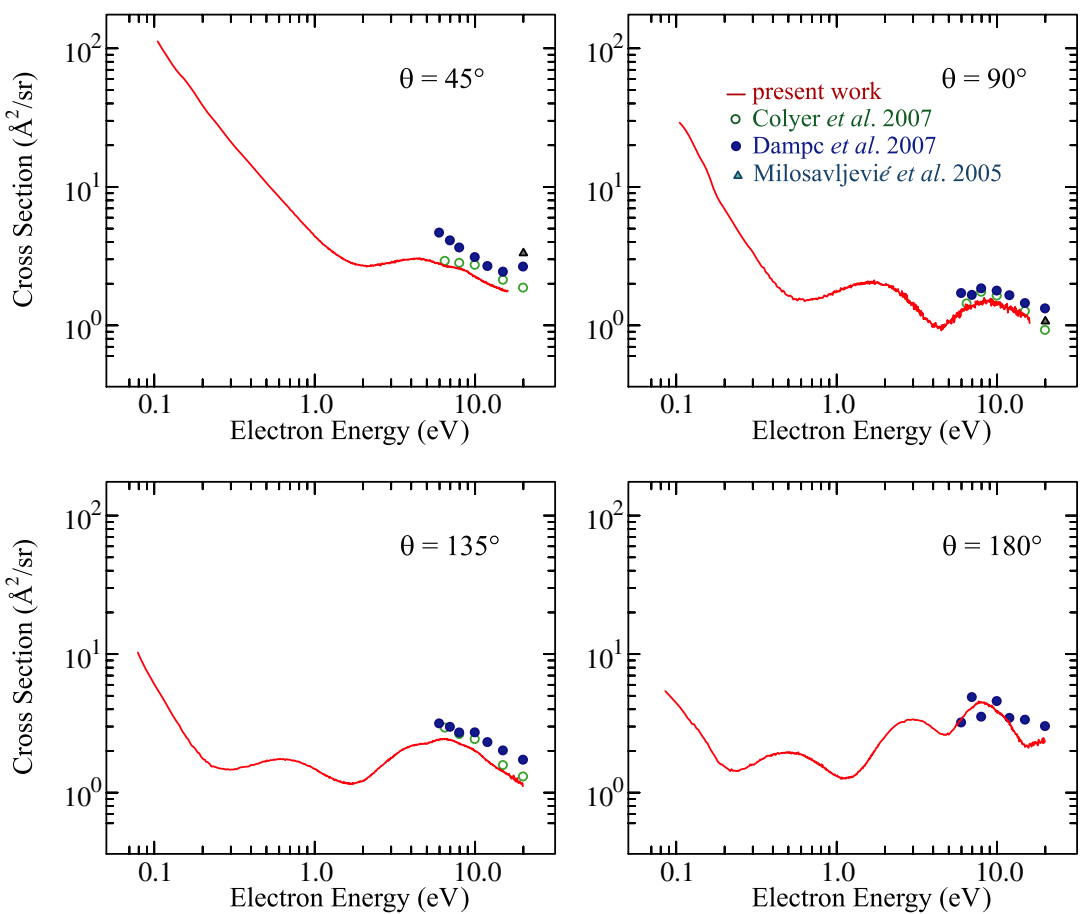

Figure 2. Elastic cross sections shown as a function of energy-comparison with the experiments of Colyer et al [15], Dampc et al [16] and Milosavljević et al [14]. The arithmetic averages of the $40^{\circ}$ and $50^{\circ}$ data are taken for all three references to compare with the present $45^{\circ}$ results, the average of $130^{\circ}$ and $140^{\circ}$ of Dampc et al [16] and the data at $130^{\circ}$ alone of Colyer et al [15] are compared with the present $135^{\circ}$ data.

values measured at $10^{\circ}, 45^{\circ}, 90^{\circ}, 135^{\circ}$ and $180^{\circ}$, as described in [22]. The angle was incremented in steps of $2.5^{\circ}$.

\section{Results and discussion}

\subsection{Differential elastic cross section}

Figure 2 shows the elastic cross sections as a function of energy and compares them to earlier experiments. The earlier data are, unfortunately, not always available at the same angles and energies. The present data at $45^{\circ}\left(135^{\circ}\right)$ are therefore compared to the average of the $40^{\circ}$ and $50^{\circ}\left(130^{\circ}\right.$ and $\left.140^{\circ}\right)$ data. The angular distributions (see below) are often curved upwards in these angular ranges and the true cross section is thus slightly less than the average of the neighbouring values - taking this into account would slightly improve the agreement at $45^{\circ}$ and $135^{\circ}$ in figure 2 . The agreement is generally within the combined confidence limits, but the present data are in most cases lower, the difference being less for the results of Colyer et al [15] than for Dampc et al [16].

Figure 3 compares the present experiment with results of calculations. The general agreement is good, both quantitatively, and in the sense that the calculations reproduce correctly all qualitative features above about $1 \mathrm{eV}$. The experimental cross sections rise steeply at low energies, particularly in the forward direction, because of the 1.6 D dipole moment of THF. This effect was excluded in the calculation of Winstead and McKoy [19], but the calculation of Trevisan et al [18] reproduces this rise at low energies qualitatively correctly. 

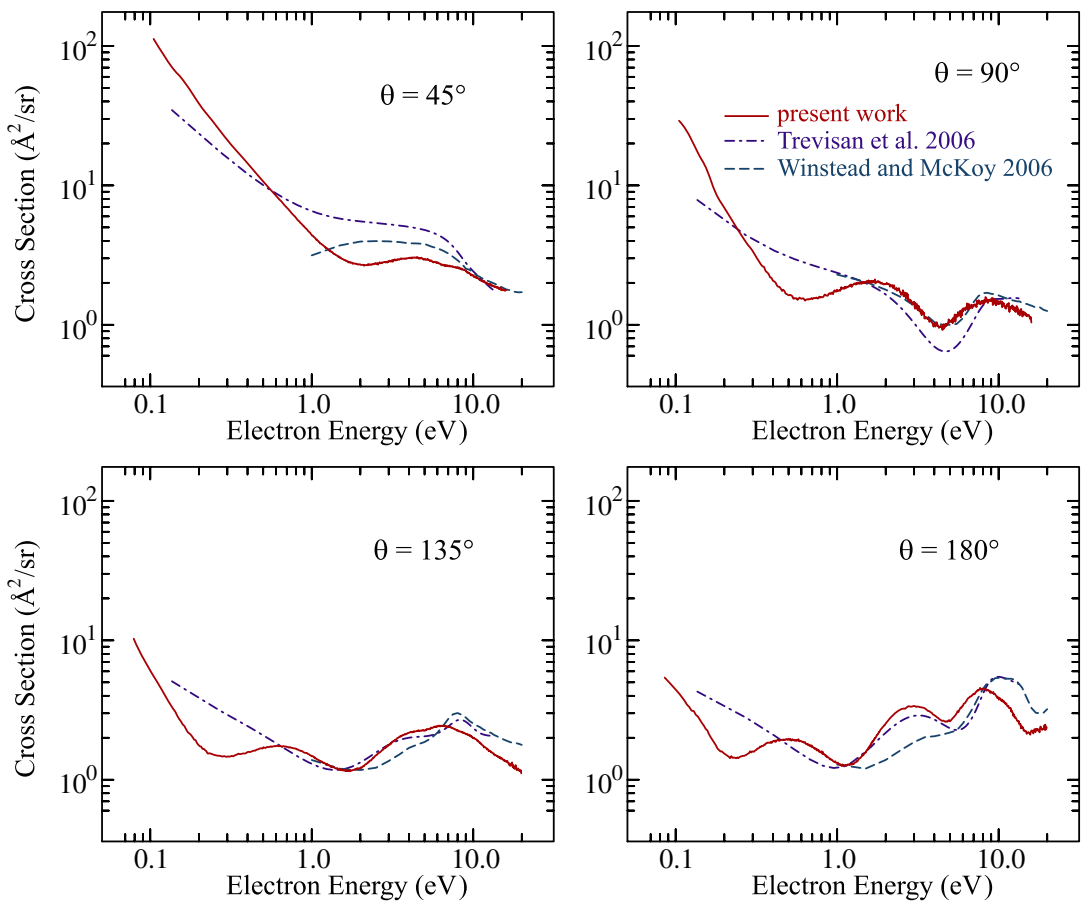

Figure 3. Elastic cross sections shown as a function of energy-comparison with the calculations of Trevisan et al [18] and Winstead and McKoy [19].

The cross sections exhibit a number of wavy structures, particularly at large scattering angles. The first minimum is found at all scattering angles and it shifts to lower energies with increasing scattering angle, from $2.1 \mathrm{eV}$ at $45^{\circ}$ to $0.24 \mathrm{eV}$ at $180^{\circ}$. It is presumably of the Ramsauer-Townsend origin. Additional minima appear at higher energies at larger scattering angles and also progressively move to lower energies with increasing scattering angle. They are obviously not of resonant origin and cannot be interpreted as resonances. Similar structures were observed at large scattering angles also in methane and neopentane [31] and appear to be a general phenomenon in near-spherical molecules.

Figure 4 shows the elastic cross sections as a function of scattering angle and compares them with earlier experiments. Again, the earlier data are not always available at the same energies and the $6.5 \mathrm{eV}$ cross section of Colyer et al [15] is compared with the present $6 \mathrm{eV}$ data. Despite this limitation, the present data agree very well with that of Colyer et al. The agreement is on the edge of the combined error limits for the data of Dampc et al [16], particularly at 6 and $20 \mathrm{eV}$. The data of Milosavljević et al [14] at $20 \mathrm{eV}$ agree well with that of Dampc et al and are somewhat higher than the present results. All four data sets agree well on the shape of the cross sections.

Finally, the calculated angular distributions are compared to the present data in figure 5 . The agreement is generally very satisfactory, both in terms of the absolute values and in terms of shapes. Thus, the broad dip in the cross section around $100^{\circ}$ at $2 \mathrm{eV}$, the two dips around $85^{\circ}$ and $150^{\circ}$ at 6 and $10 \mathrm{eV}$ are well reproduced. The remaining differences are presumably due, in part, to the fact that the resonances are generally calculated at higher energies than observed (section 3.2). Thus the (resonant) shallow wavy structures calculated by Trevisan et al [18] between $50^{\circ}$ and $180^{\circ}$ at $20 \mathrm{eV}$ are not observed in the experiment and this could be a consequence of overestimation of the resonant character at $20 \mathrm{eV}$ by the theory, the 

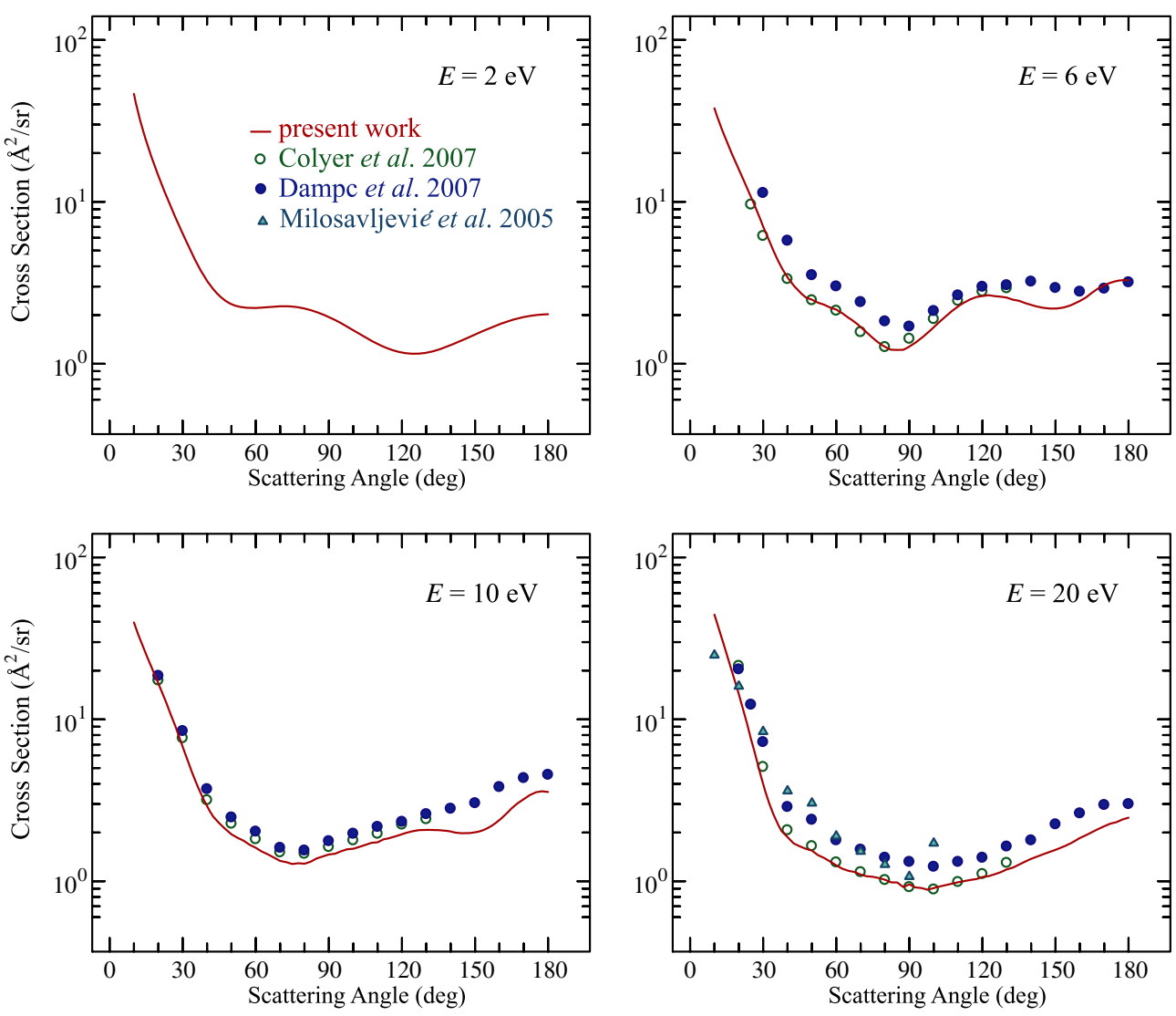

Figure 4. Elastic experimental cross sections shown as a function of scattering angle-comparison with the experiments of Colyer et al [15], Dampc et al [16] and Milosavljević et al [14]. The upper right panel shows the $6.5 \mathrm{eV}$ (and not $6 \mathrm{eV}$ ) cross section of Colyer et al [15].

resonances being at slightly lower energies in reality. In line with the conclusion already made in connection with figure 3, the calculations of Winstead and McKoy [19] underestimate the cross section at low angles and low energies where long-range scattering by the dipole field becomes important.

\subsection{Vibrational excitation cross sections}

Figure 6 shows a sample electron energy loss spectrum. It is similar to the thin-film spectrum of Lepage et al [4] and Jäggle et al [7] and the gas-phase spectrum of Milosavljević et al [14]. The individual vibrations are only partially resolved, but the consultation of the spectroscopic vibrational levels, the table of which has been given by Lepage et al [4], and of the results of routine density functional theory (DFT) normal mode calculations [32, 33], reveals that various types of vibrations have their characteristic energy ranges and can be studied individually. As indicated in the figure, the shoulder at $83 \mathrm{meV}$ is due nearly exclusively to the ring-bend vibration $v_{16}$. It is followed by a group of $\mathrm{C}-\mathrm{C}$ stretch and $\mathrm{CH}_{2}$ rock vibrations, out of which the symmetric $\mathrm{C}-\mathrm{C}$ stretch vibrations $v_{14}(109 \mathrm{meV})$ and $v_{13}(114 \mathrm{meV})$ appear to be dominant. The shoulder around $180 \mathrm{meV}$ appears to be due to a group of vibrations with a $\mathrm{CH}_{2}$ scissoring character - the symmetric vibrations $v_{6}(183 \mathrm{meV})$ and $v_{5}(187 \mathrm{meV})$ and possibly also the 

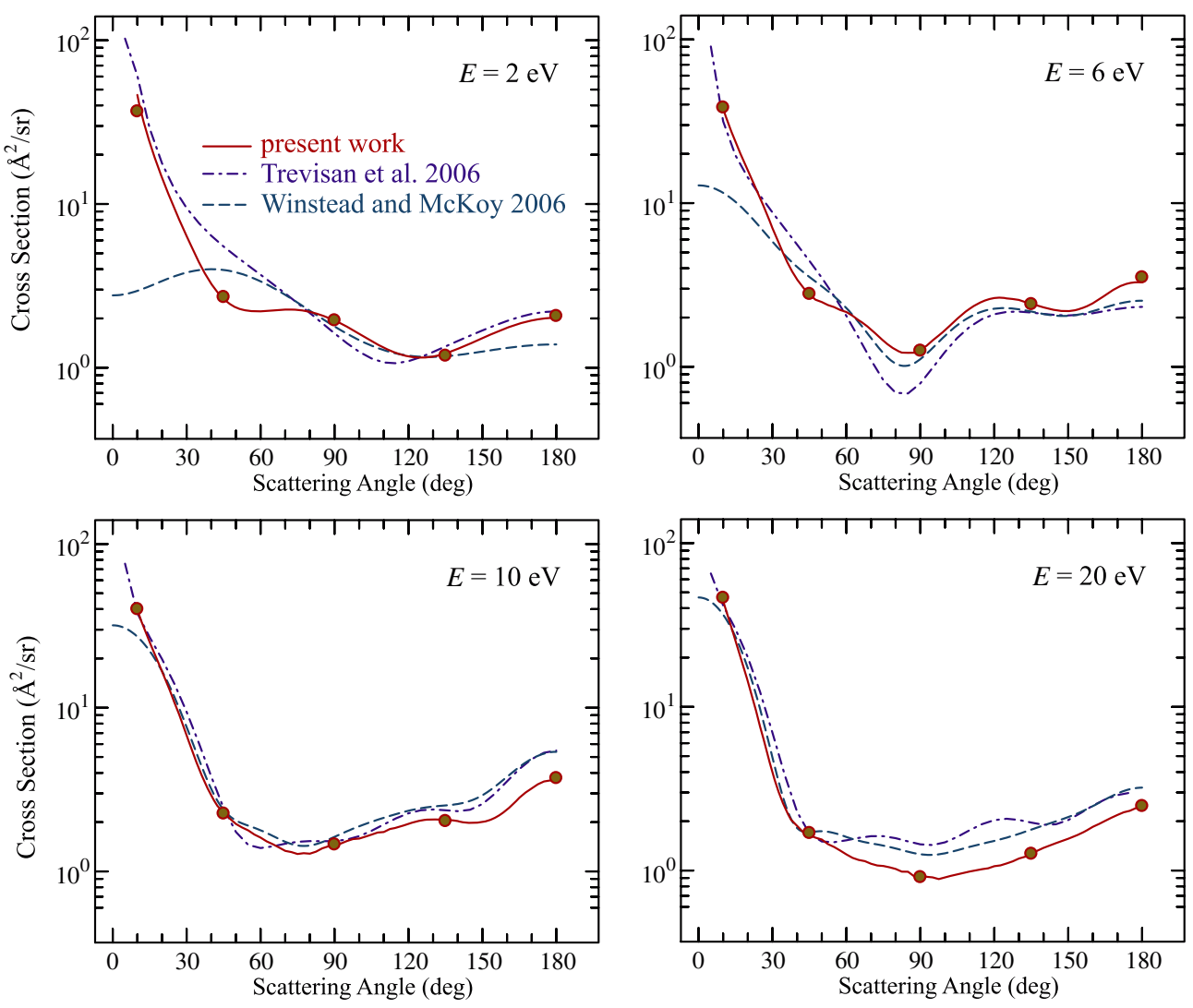

Figure 5. Elastic experimental cross sections shown as a function of scattering angle-comparison with the calculations of Trevisan et al [18] and Winstead and McKoy [19]. The filled circles show the results of the present discrete absolute measurements to which the solid lines-magnetic angular scans in $2.5^{\circ}$ increments-were fitted.

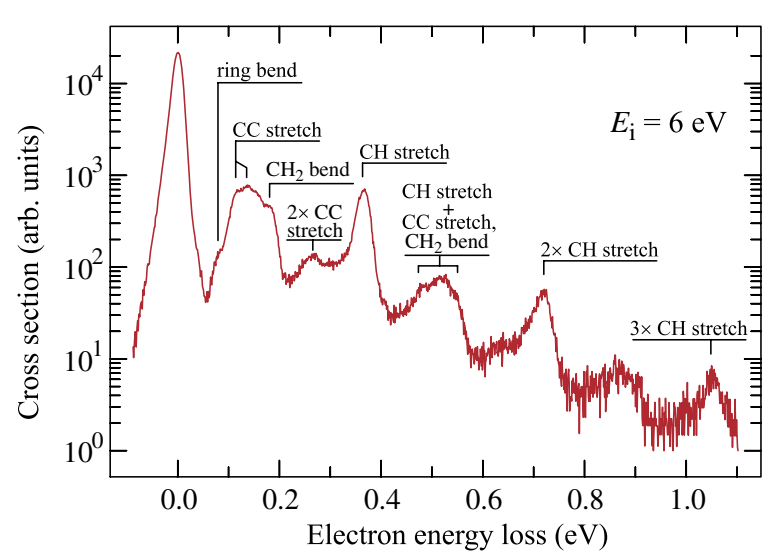

Figure 6. An electron energy loss spectrum recorded at an incident energy of $6 \mathrm{eV}$ and a scattering angle of $135^{\circ}$. 

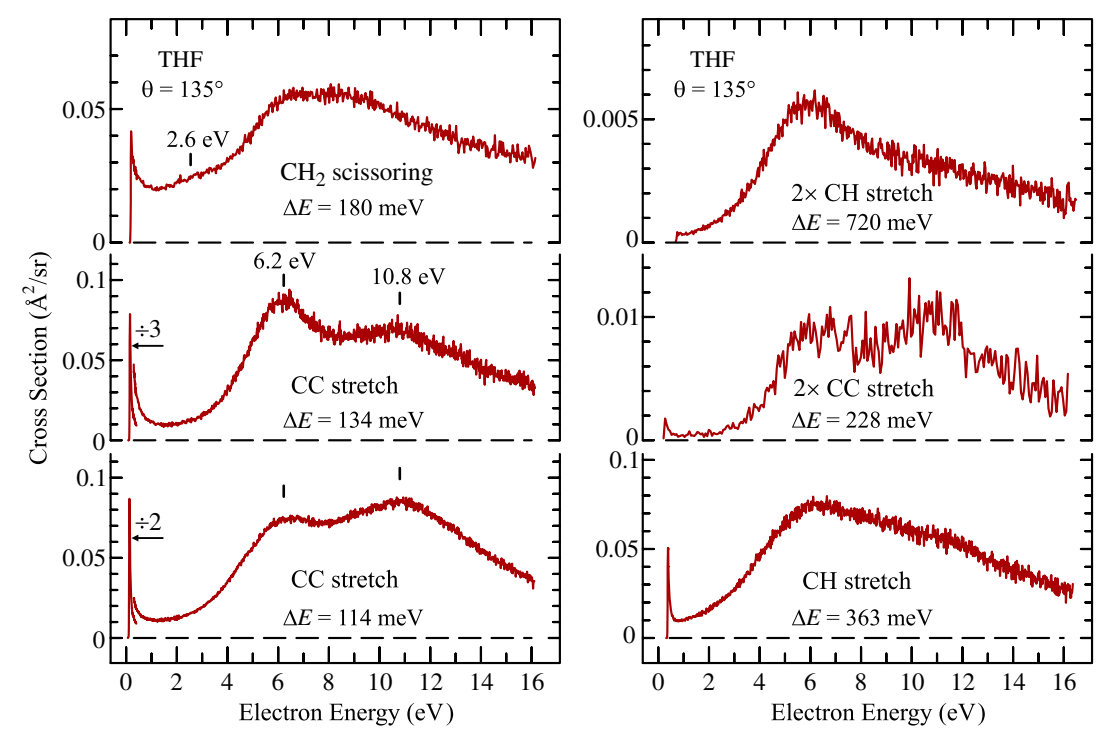

Figure 7. Cross sections for vibrational excitation.

antisymmetric vibrations $\nu_{23}(179 \mathrm{meV})$ and $\nu_{22}(185 \mathrm{meV})$. The peak around $363 \mathrm{meV}$ is due to a group of $\mathrm{C}-\mathrm{H}$ stretch vibrations. Excitation of two and three quanta of the $\mathrm{C}-\mathrm{H}$ stretch vibrations is clearly visible, together with two broad bands of combination vibrations. The appearance of overtones is an indication of resonant vibrational excitation (as opposed to dipole excitation) at $6 \mathrm{eV}$. On the other hand, the rapidly decreasing intensity of the overtones (about a factor of 10 for each $\mathrm{C}-\mathrm{H}$ stretch quantum) indicates a large autodetachment width of the resonances.

Figure 7 shows the cross sections for the selected energy losses, recorded at $135^{\circ}$. Cross sections recorded at $45^{\circ}, 90^{\circ}$ and $180^{\circ}$ were not qualitatively different in terms of shape and the resonant structure revealed, and only the $135^{\circ}$ cross sections will therefore be discussed here. The assignment and the absolute magnitudes are only indicative because of the overlap of the vibrational peaks. All fundamental mode cross sections have a threshold peak, which is (nearly) absent for the overtone excitation. The $\mathrm{C}-\mathrm{C}$ excitation cross sections have two broad bands at 6.2 and $10.8 \mathrm{eV}$. Both shape (one particle) resonances and core-excited (one hole, two particles) Feshbach resonances are expected in this energy range, but the bands in figure 7 can be assigned to shape resonances because Feshbach resonances generally affect vibrational excitation only very weakly. An additional indicator for an assignment to shape resonances rather than Feshbach resonances is the fact that both bands are substantially broader $(\sim 3 \mathrm{eV})$ than the Rydberg bands $(\sim 0.4 \mathrm{eV})$ [34], which would be the parent states of the Feshbach resonances. The shapes of the curves in figure 7 resemble the shapes of the vibrational excitation functions reported by Lepage et al [4] for the condensed THF-both exhibit two very broad bands - although the bands of the condensed THF are at lower energies by about $2 \mathrm{eV}$. Dampc et al [17] reported the $\mathrm{C}-\mathrm{H}$ stretch excitation cross section measured at $80^{\circ}$ which has a similar general shape to the present $135^{\circ}$ cross section in terms of a very broad band in the 4-14 eV range, but where three shallow bands at 6, 7.9 and $10.3 \mathrm{eV}$ can be recognized. The first and the last of these bands agree well with the present findings. No clearly distinguishable band appears at $7.9 \mathrm{eV}$ in the present $\mathrm{C}-\mathrm{H}$ stretch data in figure 7 , but the present data are not incompatible with a resonance at $7.9 \mathrm{eV}$-it could be responsible for the absence of a clear dip at $8 \mathrm{eV}$ in the $\mathrm{C}-\mathrm{H}$ stretch excitation cross section. 
C-C stretch

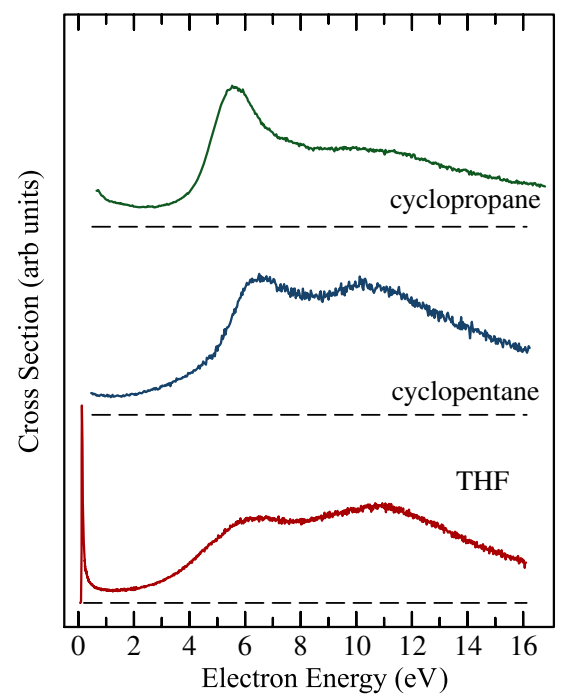

$\mathrm{CH}_{2}$ scissoring

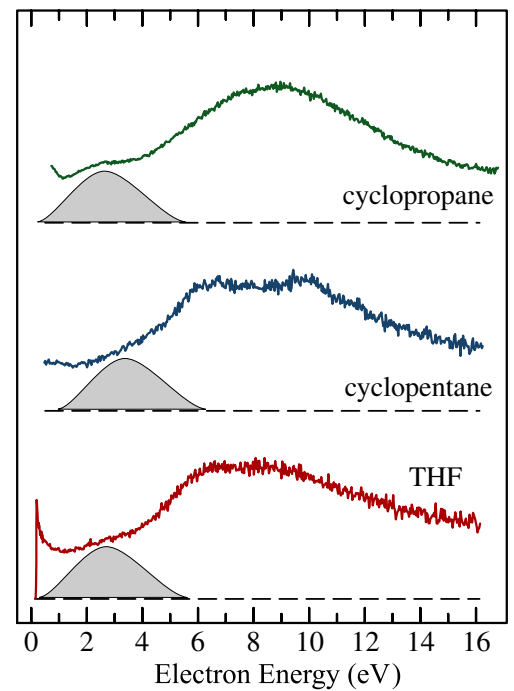

Figure 8. Comparison of the cross sections for the excitation of vibrations consisting primarily of the $\mathrm{C}-\mathrm{C}$ stretch (left) and the $\mathrm{CH}_{2}$ scissoring (right) motion for cyclopropane, cyclopentane (both from [35]) and tetrahydrofuran (the present work). The grey bell-shaped curves on the right illustrate schematically which part of the observed signal is attributed to the $\sim 2.6 \mathrm{eV}$ resonance.

The present results are in qualitative agreement with the ab initio calculation of Trevisan et al [18] who reported a $\sim 3 \mathrm{eV}$ wide resonant band at $8.6 \mathrm{eV}$ in their momentum transfer cross section, which was shown to be the result of two overlapping shape resonances of $A_{1}$ and $B_{2}$ symmetry, respectively. The two resonances were found to have very different sensitivities to target relaxation so that it is possible that their real energies are more separated than the calculation indicates and correspond to the two bands observed in figure 7 . The calculation of Winstead and McKoy [19] yielded two shape resonances at about 10 and $15 \mathrm{eV}$ in the staticexchange approximation, which shifted downwards to about 8.3 and $13 \mathrm{eV}$ when polarization effects were included - in good agreement with the present findings, if it is assumed that full inclusion of target relaxation would shift the bands to even lower energies. Shape resonances at 8.6 and $14.1 \mathrm{eV}$ were calculated also by Tonzani et al [21].

The question arises whether the DEA bands $[9,11]$ are due to the same resonances seen in the present VE cross sections or to Feshbach resonances. Aflatooni et al [11] reported two DEA bands, at 6.2 and $8 \mathrm{eV}$, of which the first agrees well with our first band in terms of energy. The DEA band is narrower, however, and we therefore favour the interpretation of the DEA bands being due to Feshbach resonances and the VE bands to shape resonances.

A closer inspection of the spectra in figure 7 reveals a third more subtle feature. The cross section for the excitation of the $\mathrm{CH}_{2}$ scissoring vibration has a substantial value in the 1.5$3 \mathrm{eV}$ range whereas the cross sections for the excitation of the other vibrations are very low in this energy range. This leads to the postulation of a third, low-lying, shape resonance centred at about $2.6 \mathrm{eV}$.

Figure 8 points out the close similarity of the resonant structure of THF to those reported earlier [35, 36] for cyclopentane and cyclopropane, and also ethylene oxide (not shown here). These studies identified a relatively narrow ${ }^{2} A_{2}^{\prime} \sigma^{*}$ shape resonance at $5.5 \mathrm{eV}$ in cyclopropane, seen in the $\mathrm{C}-\mathrm{C}$ stretch excitation in figure 8 . This resonance was much narrower 
Table 1. Integral elastic cross sections (in $\AA^{2}$ ).

\begin{tabular}{rlllllll}
\hline & \multicolumn{3}{c}{ Experiment } & & \multicolumn{3}{c}{ Theory } \\
\cline { 2 - 3 }$E(\mathrm{eV})$ & Present & {$[15]$} & {$[16]$} & & {$[19]$} & {$[21]$} & {$[20]^{\mathrm{a}}$} \\
\hline 2.0 & 40.4 & - & - & 28 & 40 & 120 \\
6.0 & 42.1 & $41.0^{\mathrm{b}}$ & 49.1 & & 32 & 35 & 65 \\
10.0 & 39.4 & 41.0 & 44.6 & & 41 & 44 & 60 \\
20.0 & 30.9 & 37.7 & 41.0 & 35 & 36 & \\
\hline
\end{tabular}

${ }^{a}$ With Born correction.

${ }^{\mathrm{b}}$ At $6.5 \mathrm{eV}$.

than its counterpart in $n$-propane because of 'symmetry narrowing' - the high symmetry of cyclopropane eliminated coupling to low partial waves and made the $f$-wave the leading wave - thus reducing the autodetachment width. This ${ }^{2} A_{2}^{\prime}$ resonance was fully reproduced by the calculation of Čurík and Gianturco [37]. This resonance was broader but clearly present in ethylene oxide-indicating that it persists even when a $\mathrm{CH}_{2}$ group is replaced by oxygen. A broader but otherwise similar resonance is found also in cyclopentane and, in the present study, in THF. The $10.8 \mathrm{eV}$ resonance in THF also has a close analogy in cyclopentane.

The similarity extends even to the $2-3 \mathrm{eV}$ resonance as illustrated on the right side of figure 8. An enhancement of the vibrational cross section in cyclopropane around $2.6 \mathrm{eV}$, selectively observed only in the cross section for the excitation of the $\mathrm{CH}_{2}$ scissoring vibration, was interpreted as an indication of a low-lying ${ }^{2} A_{2}^{\prime \prime}$ shape resonance with a large autodetachment width $[35,36]$. The assignment was based on the selectivity of the excitation of the $\mathrm{CH}_{2}$ scissoring vibration, compatible with the $\mathrm{H}-\mathrm{H}$ antibonding character of the $a_{2}^{\prime \prime}$ orbital (shown schematically in $[35,36]$ ), and on the correct prediction of the vertical attachment energy by the scaled Koopmans theorem [35,36]. A similar orbital ( $B_{2}$ in $C_{2 v}, B$ in $C_{2}, A^{\prime}$ in $C_{S}$ ), with a $\mathrm{H}-\mathrm{H}$ antibonding character and the correct scaled Koopmans theorem energy is calculated also in cyclopentane and THF. A possible alternative to this assignment has been presented by Čurík and Gianturco [37], who in their calculation obtained a broad shape resonance around $3.7 \mathrm{eV}$ in cyclopropane, but it had $A_{1}^{\prime}$ symmetry.

It may be objected that a shape resonance cannot be postulated at $2.6 \mathrm{eV}$ in THF because it has not appeared in theoretical calculations - but the observation of the enhancement of the $\mathrm{CH}_{2}$ scissoring vibration in the 2-3 energy range is an experimental fact beyond any reasonable doubt, and it is hard to imagine a mechanism for it other than a resonance. Interestingly, a shoulder around $3 \mathrm{eV}$ appears in the momentum-transfer cross section of Trevisan et al [18], although it was not explicitly interpreted as a shape resonance in the accompanying text.

The VE cross sections have also been measured as a function of scattering angle. The results are compatible with those recently reported by Dampc et al [17], with a rise in the forward and the backward direction and it is not necessary to show them here.

\subsection{Angle-integrated cross sections}

Tables 1 and 2 list the integral and momentum-transfer cross sections obtained by suitable integration of the differential cross sections (visually extrapolated between $0^{\circ}$ and $10^{\circ}$ ). The results agree well with previous measurements at 6 and $10 \mathrm{eV}$, in particular that of Colyer et al [15]. The present cross sections are somewhat lower at $20 \mathrm{eV}$. Calculated cross sections were also included in the comparison, although it could be a matter of debate whether the cross sections calculated at a fixed geometry should be compared to the experimental vibrationally elastic cross sections, or better to the electronically elastic cross sections, that is the sum of the vibrationally elastic and all vibrationally inelastic cross sections. Despite this uncertainty, the 
Table 2. Elastic momentum transfer cross sections (in $\AA^{2}$ ).

\begin{tabular}{cllllll}
\hline & \multicolumn{3}{c}{ Experiment } & & \multicolumn{2}{c}{ Theory } \\
\cline { 2 - 3 } \cline { 7 - 7 }$E(\mathrm{eV})$ & Present & {$[15]$} & {$[16]$} & & {$[19]$} & {$[18]$} \\
\hline 2.0 & 21.3 & - & - & & 21 & 23.2 \\
6.0 & 27.7 & $29.3^{\text {a }}$ & 34.6 & & 24.5 & 23.2 \\
10.0 & 24.3 & 29.7 & 31.4 & & 30 & 28.4 \\
20.0 & 16.0 & 19.2 & 22.4 & & 21.5 & \\
\hline
\end{tabular}

a At $6.5 \mathrm{eV}$

Table 3. Elastic, VE summed over all vibrational modes, and total cross sections (in $\AA^{2}$ ).

\begin{tabular}{rllllll}
\hline$E(\mathrm{eV})$ & Elastic & VE & Total & {$[12]$} & {$[38]^{\mathrm{a}}$} & {$[13]$} \\
\hline 6.0 & 42.1 & 6.6 & 48.7 & 36.4 & 41.6 & 52.0 \\
10.0 & 39.4 & 5.6 & 45.0 & 34.9 & 40.4 & 49.8
\end{tabular}

${ }^{a}$ Reference [12], corrected for incomplete rejection of forward scattered electrons [38].

calculated momentum transfer cross sections agree well with the experiments. The agreement is less satisfactory for the integral cross sections, presumably because of uncertainty in the Born correction, which yields very large cross sections in the forward direction-such electrons are not detected as scattered in the experiment.

Table 3 lists the integral VE and total (elastic $+\mathrm{VE}$ ) cross sections. Integral VE cross sections summed over all vibrational modes were obtained by recording energy-loss spectra similar to that in figure 6 at incident energies of 6 and $10 \mathrm{eV}$ and the scattering angles of $15^{\circ}$, $45^{\circ}, 90^{\circ}, 135^{\circ}$ and $180^{\circ}$, integrating under the entire vibrationally inelastic signal, and then integrating over all scattering angles. The results are very well compatible with the value of $5.5 \AA^{2}$ obtained by Dampc et al [17] at $7 \mathrm{eV}$.

The total cross sections obtained as the sum of the elastic and VE cross sections are about $10 \%$ lower than the total cross sections of Możejko et al [13]—a satisfactory agreement in view of the fact that the present cross section does not include electronic excitation, the uncertainty of the visual extrapolation below $10^{\circ}$, and the experimental difficulty of separating unscattered electrons and electrons scattered into a nearly forward direction in the transmission experiment. The present values are substantially higher than the original values of Zecca et al [12], but it has been recognized that these values were too low because of insufficient rejection of electrons scattered under small angles [38]. The value retrospectively corrected for this effect [38] is much closer to the present result.

\section{Conclusions}

THF, chosen for its relevance as a model for the sugar unit of DNA, is an interesting case for testing both the experimental and the theoretical methods for electron-molecule scattering on a relatively large and polar molecule. The challenges from the experimental point of view are that the adsorption of the molecules on the inner surfaces of the gas inlet manifold may interfere with the gas flow determination, and the large size of the molecule makes the problem of gas beam profile variations more critical. In view of these problems the agreement of the present data with earlier experiments is satisfactory. Theory appears fundamentally capable of describing the elastic scattering at energies above about $1 \mathrm{eV}$, in some cases with an impressive capacity to reproduce wavy structures in the angular distributions. Problems are larger at small scattering angles and low energies. 
Interesting features appear in the elastic cross sections recorded as a function of electron energy. The Ramsauer-Townsend minimum is observed at an energy which decreases with the scattering angle and becomes $0.24 \mathrm{eV}$ at $180^{\circ}$. Additional minima appear in the cross section at large scattering angles, similar to those reported previously for methane and neopentane. These minima are properly accounted for by the theory, indicating that they are fundamentally understood, but it would be interesting to understand their qualitative physical origin.

The momentum transfer cross sections derived from the differential cross sections are in good agreement with previous experiments and with theoretical predictions. The agreement with theory is in some cases less satisfactory for the integral cross sections, presumably because the experiment is not capable of measuring the large cross sections for scattering into very small angles predicted by the Born correction. Comparison of differential cross sections is a less problematic method of testing theory in this respect. The present total (elastic + VE) cross sections are in a good agreement with the total cross sections measured by the beam attenuation (transmission) method.

Shape resonances are best revealed by VE cross sections. Two resonant bands were observed at 6.2 and $10.8 \mathrm{eV}$, in agreement with other recent experimental works and in qualitative agreement with theory. Comparison of the present results with earlier results on cyclopropane, ethylene oxide, and cyclopentane reveals many common features and a close resemblance of the resonant structure of cyclic hydrocarbons, which is preserved even when one of the $\mathrm{CH}_{2}$ groups is replaced by an oxygen atom. This resemblance extends even to the $\sim 2.6 \mathrm{eV}$ resonance originally observed in cyclopropane, ethylene oxide and cyclopentane. This resonance might be important as a relay state in electron transfer through the sugar unit of DNA.

\section{Acknowledgments}

I thank Cynthia Trevisan, Thomas Rescigno, Carl Winstead, Vincent McKoy and Jimena Gorfinkiel for providing their calculated cross sections in a numerical form and helpful comments. This research is part of project no 200020-113599/1 of the Swiss National Science Foundation.

\section{References}

[1] Boudaiffa B, Cloutier P, Hunting D, Huels M A and Sanche L 2000 Science 2871658

[2] Martin F, Burrow P D, Cai Z, Cloutier P, Hunting D and Sanche L 2004 Phys. Rev. Lett. 93068101

[3] Antic D, Parenteau L, Lepage M and Sanche L 1999 J. Phys. Chem. B 1036611

[4] Lepage M, Letarte S, Michaud M, Motte-Tollet F, Hubin-Franskin M J, Roy D and Sanche L 1998 J. Chem. Phys. 1095980

[5] Antic D, Parenteau L and Sanche L 2000 J. Phys. Chem. B 1044711

[6] Breton S P, Michaud M, Jäggle C, Swiderek P and Sanche L 2004 J. Chem. Phys. 12111240

[7] Jäggle C, Swiderek P, Breton S P, Michaud M and Sanche L 2006 J. Phys. Chem. B 11012512

[8] Park Y S, Cho H, Parenteau L, Bass A D and Sanche L 2006 J. Chem. Phys. 125074714

[9] Sulzer P et al 2006 J. Chem. Phys. 125044304

[10] Ibănescu B C, May O and Allan M 2007 in preparation

[11] Aflatooni A, Scheer A M and Burrow P D 2006 J. Chem. Phys. 125054301

[12] Zecca A, Perazzolli C and Brunger M 2005 J. Phys. B: At. Mol. Opt. Phys. 382079

[13] Możejko P, Ptasińska-Denga E, Domaracka A and Szmytkowski C 2006 Phys. Rev. A 74012708

[14] Milosavljević A R, Giuliani A, Šević D, Hubin-Franskin M J and Marinković B P 2005 Eur. Phys. J. D 35411

[15] Colyer C J, Vizcaino V, Sullivan J P, Brunger M J and Buckman S J 2007 New J. Phys. 941

[16] Dampc M, Milosavljević A R, Linert I, Marinković B P and Zubek M 2007 Phys. Rev. A 75042710

[17] Dampc M, Linert I, Milosavljević A R and Zubek M 2007 Chem. Phys. Lett. 44317

[18] Trevisan C S, Orel A E and Rescigno T N 2006 J. Phys. B: At. Mol. Opt. Phys. 39 L255 
[19] Winstead C and McKo y V 2006 J. Chem. Phys. 125074302

[20] Bouchiha D, Gorfinkiel J D, Caron L G and Sanche L 2006 J. Phys. B: At. Mol. Opt. Phys. 39975

[21] Tonzani S and Greene C H 2006 J. Chem. Phys. 125094504

[22] Allan M 2005 J. Phys. B: At. Mol. Opt. Phys. 383655

[23] Allan M 1995 J. Phys. B: At. Mol. Opt. Phys. 285163

[24] Gopalan A, Bömmels J, Götte S, Landwehr A, Franz K, Ruf M W, Hotop H and Bartschat K 2003 Eur. Phys. $J$. D 2217

[25] Nickel J C, Zetner P W, Shen G and Trajmar S 1989 J. Phys. E: Sci. Instrum. 22730

[26] Nesbet R K 1979 Phys. Rev. A 2058

[27] Buckman S J, Gulley R J, Moghbelalhossein M and Bennett S J 1993 Meas. Sci. Technol. 41143

[28] Khakoo M A, Keane K, Campbell C, Guzman N and Hazlett K 2007 J. Phys. B: At. Mol. Opt. Phys. 40 at press

[29] Read F H and Channing J M 1996 Rev. Sci. Instrum. 672373

[30] Zubek M, Gulley N, King G C and Read F H 1996 J. Phys. B: At. Mol. Opt. Phys. 29 L239

[31] Allan M 2007 Atomic and molecular data and their applications AIP Conf. Proc. vol 91 ed E Roueff (New York: American Institute of Physics) p 107

[32] Granovsky A A 2007 PC GAMESS version 6.4 http://classic.chem.msu.su/gran/gamess/index.html

[33] Schmidt M W et al 1993 J. Comput. Chem. 141347

[34] Bremner L J, Curtis M G and Walker I C 1991 J. Chem. Soc. Faraday Trans. 871049

[35] Allan M and Andrić L 1996 J. Chem. Phys. 1053559

[36] Allan M 1993 J. Am. Chem. Soc. 1156418

[37] Čurík R and Gianturco F A 2002 J. Phys. B: At. Mol. Opt. Phys. 351235

[38] Brunger M J 2007 private communication 